\title{
RENDIMENTOS DO PROCESSAMENTO E COMPOSIÇÃO CENTESIMAL DE FILÉS DO JUNDIÁ Rhamdia vouleżi
}

\author{
PROCESSING YELD AND PROXIMATE COMPOSITION OF Rhamdia \\ voulezi FILLETS
}

\author{
Elenice Souza dos Reis Goes ${ }^{1}$ \\ Aldi Feiden ${ }^{2}$ \\ Dacley Hertes $\mathrm{Neu}^{3}$ \\ Marcio Douglas Goes ${ }^{4}$ \\ Wilson Rogério Boscolo ${ }^{2}$ \\ Altevir Signor ${ }^{2}$
}

\begin{abstract}
${ }^{1}$ Universidade Estadual de Maringá, Centro de Ciências Agrárias, Programa de Pós-Graduação em Ciência de Alimentos, Maringá, PR, Brasil.

2Universidade Estadual do Oeste do Paraná, Centro de Engenharias e Ciências Exatas, Curso de Engenharia de Pesca, Toledo, PR, Brasil.

3Universidade Federal da Grande Dourados, Faculdade de Ciências Agrárias, Curso de Engenharia de Aquicultura, Dourados, MS, Brasil.

"Universidade Federal do Paraná, Programa de Pós-Graduação em Aquicultura e Desenvolvimento Sustentável, Palotina, PR, Brasil.

*Autor para correspondência: elenicesreis@yahoo.com.br
\end{abstract}

\section{Resumo}

O objetivo deste trabalho foi avaliar o efeito do peso de abate e do sexo sobre os rendimentos do processamento e sobre a composição centesimal do jundiá ( $R$. voulezi). Foram calculados os rendimentos percentuais em relação à massa do peixe inteiro (rendimentos da carcaça, tronco limpo, músculo abdominal, filé, partes comestíveis, cabeça, pele, gônadas e gordura visceral). Não houve diferença estatística nos rendimentos entre as classes $(\mathrm{P}>0,05)$. Entretanto, ao se avaliarem os rendimentos entre os sexos, foi observada diferença significativa $(\mathrm{P}<0,05)$ para os rendimentos da carcaça, tronco limpo, músculo abdominal e gordura visceral, sendo que os machos obtiveram os maiores valores nestes itens. As fêmeas apresentaram maior $(\mathrm{P}<0,05)$ rendimento das gônadas. Os teores de proteína bruta, cinzas e carboidratos não diferiram $(\mathrm{P}>0,05)$ entre as classes, porém, a umidade, lipídeos e valor calórico tiveram diferença significativa $(\mathrm{P}<0,05)$ entre as classes, sendo que a umidade diminuiu com o aumento do tamanho dos peixes e o teor de lipídeos e valor calórico foi maior nos peixes da classe 301-400 g. Concluiu-se que os rendimentos do processamento do jundiá (R. voulezi) com massa entre 100 e 400 g são iguais, sendo que os peixes de 301 a $400 \mathrm{~g}$ apresentam maior teor de lipídeos no filé.

Palavras-chave: aproveitamento industrial; peixe nativo; rendimento corporal.

\begin{abstract}
The aim of this study was evaluate the effect of slaughter weight and sex on processing yield and on the proximate composition of Rhamdia voulezi. We calculated the percentage yield of the whole fish (carcass, clean trunk, abdominal muscle, fillet, edible parts, head, skin, gonads and visceral fat yield). There was no statistical difference in yields among weight classes ( $P>0.05)$. However, when assessing the income between sexes we observed significant difference $(\mathrm{P}<0.05)$ for carcass, clean
\end{abstract}


trunk, muscle and abdominal visceral fat yield, and males presented the highest values for these items. Females had higher $(\mathrm{P}<0.05)$ gonads yield. Crude protein, ashes and carbohydrates did not differ $(\mathrm{P}>0.05)$ among the classes, however, moisture, lipids and calories had significant difference $(\mathrm{P}<0.05)$ among the classes, and humidity decreased with increasing size of the fish, and lipid content and calorific value were higher for fish weighing 301-400 $\mathrm{g}$. We concluded $R$. voulezi with mass between 100 and $400 \mathrm{~g}$ presents equal yield processing, and fish weighing 301-400 $\mathrm{g}$ have higher lipid content in the fillet.

Keywords: carcass yield; industrial use; native fish.

Enviado em: 18 novembro 2011.

Aceito em: 13 agosto 2015.

\section{Introdução}

O jundiá Rhamdia voulezi é um peixe endêmico e nativo da bacia hidrográfica do Rio Iguaçu, caracterizada por um isolamento geográfico que proporcionou um elevado grau de endemismo da sua ictiofauna ${ }^{(1)}$. O elevado desnível dessa bacia foi atrativo para a formação de usinas hidrelétricas, formando diversos reservatórios ao longo do rio, sendo estes locais propícios para o cultivo de peixes nativos em tanques-rede.

Dentre os jundiás do gênero Rhamdia, o $R$. voulezi possui potencial para aquicultura no sul do Brasil devido ao alto crescimento, excelente eficiência alimentar e resistência ao manejo, associados à carne saborosa e bem aceita pelo mercado consumidor ${ }^{(2)}$. Diversos estudos vêm sendo realizados com o intuito de proporcionar bases para o cultivo do $R$. voulezi com foco principalmente na nutrição da espécie $^{(2-7)}$.

Assim, informações sobre o processamento de espécies com potencial para piscicultura são necessários, pois dão subsídios à indústria de beneficiamento do pescado. Além disso, o processamento do pescado é primordial para a agregação de valor à matéria-prima, sendo essencial no mercado consumidor, principalmente em escala comercial ${ }^{(8)}$. O conhecimento dos valores referentes aos rendimentos do processamento auxilia as indústrias beneficiadoras de pescado a planejarem a produção, sendo efetivo para avaliar a eficiência produtiva da empresa. Conforme Galvão et al. ${ }^{(9)}$, estas informações também podem auxiliar o controle de qualidade e o sistema de rastreabilidade do pescado, aumentando a rentabilidade do processamento.

A qualidade da carcaça do pescado é um fator imprescindível para definição dos processos de preparação dos produtos e dos tipos de cortes ${ }^{(10)}$. Diversas espécies já tiveram seus rendimentos do processamento estudados e caracterizados, como a tilápia-do-Nilo (Oreochromis niloticus) ${ }^{(11)}$, matrinxã (Brycon cephalus $)^{(12)}$, piracanjuba (Brycon orbignyanus $)^{(10)}$, jundiá $($ Rhamdia quelen) ${ }^{(13,14)}$, armado (Pterodoras granulosus) ${ }^{(15)}$, surubim (Pseudoplatystoma $\left.\mathrm{sp}\right)^{(16)}$, pacu (Piaractus mesopotamicus $)^{(17)}$, viola (Loricariichthys anus) ${ }^{(18)}$ e truta arco-íris $(\text { Oncorhynchus mykiss })^{(19)}$, entre outros. Estes estudos demonstraram que a obtenção de dados do processamento de espécies com potencial de industrialização é fundamental para proporcionar informações técnicas que sirvam de base para sua utilização comercial.

Dessa forma, este estudo teve por objetivo avaliar o efeito do peso de abate e do sexo sobre os rendimentos do processamento e sobre a composição centesimal do jundiá $R$. voulezi.

\section{Material e Métodos}

Os exemplares de jundiá $R$. voulezi utilizados neste experimento foram provenientes de cultivo em tanques-rede no reservatório da Usina Hidrelétrica Governador José Richa, no Centro de Desenvolvimento e Difusão de Tecnologias do rio Iguaçu (CDT Iguaçu), localizado no município de 
Boa Vista da Aparecida - Paraná (Figura 1).

Para realização das análises de rendimentos, 90 peixes foram insensibilizados por secção da medula espinhal e transportados em gelo para o Laboratório de Tecnologia do Pescado, do Grupo de Estudos de Manejo na Aquicultura - GEMAq, da Universidade Estadual do Oeste do Paraná - Campus Toledo. As análises foram realizadas de janeiro a março de 2009. Para avaliação dos rendimentos do processamento, os animais foram separados em três classes de massa: Classe A (100-200g), Classe B (201-300g) e Classe C (301-400g). Para a aferição da massa das diferentes partes corporais, foi utilizada uma balança semi-analítica com capacidade para $4.200 \mathrm{~g}$ e precisão $0,01 \mathrm{~g}$, da marca Shimadzu.

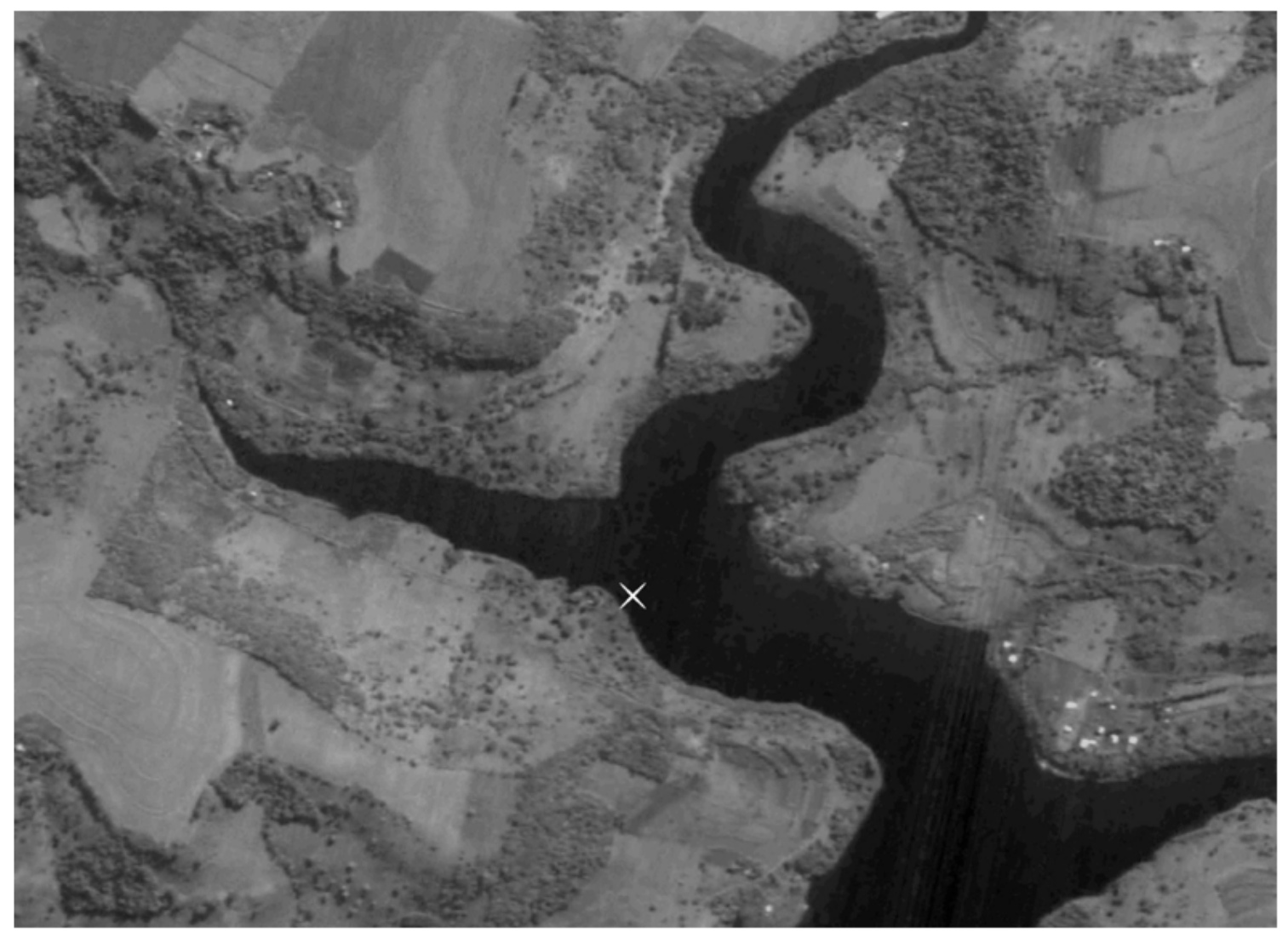

Figura 1: Localização do CDT Iguaçu. Imagem de Satélite. Fonte: Instituto Nacional de Pesquisas Espaciais $^{(20)}$.

Inicialmente, foram realizadas as medidas de massa total $(\mathrm{g})$ e comprimento total $(\mathrm{cm})$, mensurado com o auxílio de um ictiômetro. Os peixes foram então eviscerados, com posterior retirada da cabeça, couro, músculo abdominal e filetagem. Foram tomadas medidas de massa do peixe inteiro eviscerado, da cabeça, do couro, das gônadas, da gordura visceral, do músculo abdominal (barriguinha), do filé e verificação das gônadas para determinação do sexo.

A carcaça foi obtida a partir da abertura ventral da cavidade abdominal, desde o orifício urogenital até os ossos da mandíbula, seguido de retirada cuidadosa das vísceras, para evitar a contaminação da carne com o material fecal. Depois da retirada da cabeça, pele e nadadeiras, obtém-se o tronco limpo, utilizado na obtenção do músculo abdominal e filé. Com estes dados, foram calculados os rendimentos percentuais em relação à massa do peixe inteiro, obtendo-se dados de rendimento da carcaça, rendimento do tronco limpo, rendimento do músculo abdominal, rendimento do filé, rendimento das partes comestíveis, rendimento da cabeça, rendimento da pele, rendimento das gônadas e rendimento da gordura visceral. Avaliaram-se os rendimentos em relação às classes de massa e entre o sexo dos exemplares. 
A composição centesimal do filé (umidade, proteína bruta, lipídeos e cinzas) foi realizada de acordo com metodologia proposta pela $\mathrm{AOAC}^{(21)}$. O teor de carboidratos foi calculado pela diferença dos outros componentes da composição centesimal e o valor calórico total foi obtido pela soma da multiplicação dos valores das médias de proteína, lipídios e carboidratos multiplicados pelos fatores 4, 9 e 4, respectivamente ${ }^{(22)}$.

Os dados foram submetidos ao teste de homogeneidade, normalidade e análise de variância em nível de 5\%. Quando observadas diferenças significativas, foi aplicado o teste de Tukey, através do programa Statistic Analysis System (SAS, SAS Inst. Inc. Cary, NC, EUA).

\section{Resultados e Discussão}

Os jundiás $R$. voulezi da Classe A obtiveram massa e comprimento de 106,33 g e 21,54 cm, na Classe $\mathrm{B}, 241,33 \mathrm{~g}$ e 27,73 cm e na Classe C, 375,64 g e 31,42 cm. Os exemplares machos tiveram 240,10 g e $26,85 \mathrm{~cm}$, enquanto as fêmeas $243,56 \mathrm{~g}$ e $27,01 \mathrm{~cm}$ de massa e comprimento total, respectivamente. Os valores percentuais de rendimentos médios do processamento não apresentaram diferenças $(\mathrm{P}>0,05)$ entre as classes de massa avaliadas (Tabela 1$)$.

Tabela 1: Rendimentos médios (\%) dos diferentes cortes do jundiá R. voulezi separados em classes de massa (g)

\begin{tabular}{lccc}
\hline & \multicolumn{3}{c}{ Classes (g) } \\
\cline { 2 - 4 } Rendimento (\%) & $\mathbf{1 0 0 - 2 0 0}$ & $\mathbf{2 0 1 - 3 0 0}$ & $\mathbf{3 0 1 - 4 0 0}$ \\
\hline Carcaça $^{\mathrm{NS}}$ & $83,18 \pm 7,65$ & $85,44 \pm 4,64$ & $83,88 \pm 6,17$ \\
Tronco limpo $^{\mathrm{NS}}$ & $46,41 \pm 3,84$ & $47,63 \pm 4,62$ & $48,57 \pm 5,16$ \\
Músculo abdominal $^{\mathrm{NS}}$ & $5,45 \pm 1,85$ & $5,59 \pm 1,38$ & $5,02 \pm 1,75$ \\
FiléNS $^{\text {Partes comestíveis }}{ }^{\mathrm{NS}}$ & $25,09 \pm 3,40$ & $28,18 \pm 5,08$ & $29,36 \pm 3,24$ \\
Cabeça & $30,54 \pm 3,56$ & $33,46 \pm 5,84$ & $34,38 \pm 4,39$ \\
Pele $^{\mathrm{NS}}$ & $26,14 \pm 3,91$ & $26,67 \pm 2,58$ & $26,04 \pm 2,51$ \\
Gônadas $^{\mathrm{NS}}$ & $6,49 \pm 1,33$ & $6,78 \pm 1,60$ & $6,81 \pm 1,47$ \\
Gordura visceral $^{\mathrm{NS}}$ & $5,12 \pm 4,53$ & $6,62 \pm 5,46$ & $7,72 \pm 6,59$ \\
\hline
\end{tabular}

NS=Não significativo. Dados expressos em média \pm desvio padrão

O rendimento de carcaça médio foi de $84,17 \%$, valor similar aos encontrados por Melo et al. ${ }^{(23)}$, que observaram percentuais entre 80,0 e $82,5 \%$ para jundiás $R$. quelen entre 40,0 e 45,0g. Para tilápias Oreochromis niloticus entre 300-400 g, Souza e Maranhão ${ }^{(11)}$ encontraram rendimento de carcaça de 75,61\%. Em surubins Pseudoplatystoma sp. foi encontrada média de 73,31\% para carcaça sem vísceras e nadadeiras ${ }^{(16)}$.

Apesar de jundiás de 100 a 400 g não apresentarem diferenças significativas $(\mathrm{P}>0,05)$ nos rendimentos do processamento (Tabela 1), quando os animais foram separados por sexo, pôde-se observar que os rendimentos da carcaça, do tronco limpo, do músculo abdominal, das gônadas e da gordura visceral (Tabela 2) apresentaram diferença significativa $(\mathrm{P}<0,05)$ entre macho e fêmea. Somente os rendimentos do filé, das partes comestíveis, da cabeça e da pele não apresentaram diferença significativa $(\mathrm{P}>0,05)$ entre os machos e fêmeas.

$\mathrm{O}$ rendimento de carcaça dos machos foi maior $(\mathrm{P}<0,05)$ do que das fêmeas, corroborando estudo feito por Reidel et al. ${ }^{(14)}$ com jundiás ( $R$. quelen) criados em tanques rede. Os autores supracitados verificaram que o rendimento de carcaça de machos é superior ao de fêmeas de $R$. quelen, e o teor de proteína na dieta pode influenciar, quando a mesma estiver em quantidades insuficientes. 
Tabela 2: Rendimentos médios (\%) dos diferentes cortes do jundiá R. voulezi separados por sexo

\begin{tabular}{|c|c|c|}
\hline \multirow[b]{2}{*}{ Rendimento (\%) } & \multicolumn{2}{|c|}{ Sexo } \\
\hline & Macho & Fêmea \\
\hline Carcaça ${ }^{1}$ & $87,45 \pm 5,95 a$ & $80,15 \pm 6,29 b$ \\
\hline Tronco limpo ${ }^{1}$ & $49,98 \pm 4,38 \mathrm{a}$ & $44,55 \pm 5,03 \mathrm{~b}$ \\
\hline Músculo abdominal $^{1}$ & $5,80 \pm 1,52 a$ & $4,80 \pm 1,72 b$ \\
\hline FiléNs & $37,32 \pm 4,53$ & $25,92 \pm 4,62$ \\
\hline Partes comestíveis ${ }^{\mathrm{NS}}$ & $43,12 \pm 4,92$ & $33,07 \pm 5,42$ \\
\hline Cabeça ${ }^{\mathrm{NS}}$ & $26,29 \pm 3,05$ & $26,28 \pm 2,68$ \\
\hline Pele NS & $6,86 \pm 1,50$ & $6,49 \pm 1,47$ \\
\hline Gônadas ${ }^{1}$ & $3,04 \pm 4,66 \mathrm{~b}$ & $10,71 \pm 5,88 \mathrm{a}$ \\
\hline Gordura visceral $^{1}$ & $2,03 \pm 1,16 \mathrm{a}$ & $1,15 \pm 1,09 b$ \\
\hline
\end{tabular}

O rendimento de filé não apresentou diferenças significativas $(\mathrm{P}>0,05)$ entre as classes ou entre sexos, com valores entre 25,09 e $42,13 \%$. O filé é considerado o tipo de corte mais comumente comercializado tendo, portanto, vantagem quanto à aceitação pelo consumidor. Entretanto, para peixes de couro, o filé deve ser retirado de maneira distinta ao filé de tilápia, o que pode fazer com que alguns outros produtos sejam gerados, como é o caso do músculo abdominal. Como o rendimento do filé depende também da destreza do filetador, não podem ser excluídos os fatores ligados ao processo de filetagem, quando analisada a ausência de diferenças estatísticas entre os sexos ou entre as classes de massa estudadas $^{(13)}$. Carneiro et al. ${ }^{(13)}$ encontraram valores de rendimento de $29,55 \%$ para o filé de juvenis de jundiá $R$. quelen apresentando massas entre 201 a 300 g, e de 31,45\% para machos da classe 301 a $400 \mathrm{~g}$, sendo estes resultados próximos aos encontrados para o $R$. voulezi no presente estudo.

Reidel et al. ${ }^{(14)}$ encontraram rendimentos de filés em torno de 30 a $40 \%$, sendo que os machos obtiveram rendimento de filé superior. No atual estudo, essa diferença não foi visualizada, porém o rendimento encontra-se próximo ao observado pelos demais autores. Outro fato que pode estar relacionado à diminuição do rendimento de filé é o tempo de cultivo se aproximar do período reprodutivo, já que neste período as gônadas aumentam de volume e o rendimento das partes corporais diminui ${ }^{(14)}$. Contudo, não pode ser descartada a destreza do filetador, fato que pode ser determinante para o aproveitamento desse tipo de corte.

Os valores de rendimento do músculo abdominal não tiveram diferenças entre classes, porém, apresentaram diferenças estatísticas $(\mathrm{P}<0,05)$ entre os sexos, com maior rendimento para machos, de $5,80 \%$. Possivelmente os animais entraram em período reprodutivo, dessa forma as fêmeas retraíram o músculo abdominal para a formação e crescimento das gônadas, enquanto os machos não, fato comprovado pelo rendimento das gônadas entre os sexos, que também foi distinto $(\mathrm{P}<0,05)$. $\mathrm{O}$ músculo abdominal (popularmente conhecido como "barriguinha") é um produto muito apreciado e gerado a partir do processamento de muitas espécies de peixe ${ }^{(13)}$. As indústrias vendem este produto como petisco, tendo boa aceitação no mercado. Para o bagre africano Clarias gariepinus, com massa inferior a $1 \mathrm{~kg}$, Souza et al. ${ }^{(24)}$ encontraram $8,22 \%$ de rendimento do músculo abdominal, valor superior ao observado no presente estudo para o jundiá $R$. voulezi. Para jundiás $R$. quelen alimentados com diferentes óleos na ração, Losekann et al. ${ }^{(25)}$ também verificaram rendimento do músculo abdominal em torno de $8 \%$.

$\mathrm{O}$ rendimento de cabeça não foi significativo $(\mathrm{P}>0,05)$ entre as classes de massa, com valores de 26,04 a $26,67 \%$. Gomiero et al. ${ }^{(12)}$ afirmam que a relação inversa entre o rendimento da cabeça e o rendimento potencial já é comprovada. A massa da cabeça é um bom indicador do rendimento do tronco limpo, pois, à medida que o tronco vai sendo manipulado, a correlação diminui, atingindo menor valor para filé sem pele. Assim, o aprimoramento das formas de processamento introduz fatores 
independentes do tamanho da cabeça, como espessura da pele e carne retirada da coluna vertebral. Verificou-se que as fêmeas de $R$. voulezi apresentaram menores valores de rendimentos de carcaça, tronco limpo, músculo abdominal e rendimento de gordura visceral, tendo, porém, maior rendimento de gônadas, de $10,71 \%$. Conforme Carneiro et al. ${ }^{(13)}$, as gônadas das fêmeas dos jundiás podem representar mais de $10 \%$ da massa total. Assim, os menores rendimentos das fêmeas em comparação com os machos podem ser explicados pelo desvio de energia metabólica para o crescimento das gônadas em detrimento do crescimento somático. Isso fica evidenciado pelo cultivo percorrer o período reprodutivo de peixes nativos, que geralmente é de outubro a março, porém o jundiá apresenta dois picos anuais, nos meses de agosto-setembro e janeiro-fevereiro ${ }^{(26)}$.

O rendimento de gordura visceral é influenciado pelo alimento que os animais consomem, principalmente com relação aos níveis energéticos. Como os animais confinados nos tanques rede receberam dieta comercial e balanceada, não houve diferenças por entre as classes massa; no entanto, os machos em comparação com as fêmeas obtiveram maior conteúdo de gordura visceral, fato relacionado ao comportamento reprodutivo.

Em relação à composição centesimal dos filés, observou-se que a umidade e os lipídeos diferiram $(\mathrm{P}<0,05)$ entre as classes estudadas, com maiores valores de umidade para a classe de $100 \mathrm{a} 200 \mathrm{~g}$, de $77,10 \%$ (Tabela 3 ). Reidel et al. ${ }^{(14)}$ observaram que a umidade do jundiá $R$. quelen difere entre os meses do ano, contudo, os valores obtidos pelos autores são inferiores aos verificados no atual estudo. Observou-se que a umidade diminuiu concomitantemente com o aumento do tamanho dos peixes e isto pode ser explicado pelo fato de que, conforme o acréscimo no tamanho do peixe, o teor de umidade das diferentes partes do corpo diminui, enquanto teores de proteína e gordura aumentam ${ }^{(27)}$. Esta observação também foi feita por Ahmed et al. ${ }^{(28)}$, que obtiveram menor teor de umidade para exemplares menores de $500 \mathrm{~g}$ de Cirrhinus mrigala (74,9\%), em comparação com animais entre 1001-1500 g, que tiveram 71,5\% de umidade no filé.

Em pescados, o teor de umidade tem uma relação inversamente proporcional com a quantidade de gordura do mesmo ${ }^{(29,30)}$, sendo que a água é o constituinte em maior proporção. Este fato foi observado no presente trabalho, pois a classe 100 a $200 \mathrm{~g}$ teve maior umidade $(77,10 \%)$ e menor teor de lipídeos (3,18\%). Já a classe de 301 a 400 g apresentou menor valor de umidade $(73,59 \%)$ e maior nível de lipídeos $(7,73 \%)$.

Tabela 3: Composição centesimal e valor calórico de filés de $R$. voulezi em diferentes classes de massa

\begin{tabular}{lccc}
\hline & \multicolumn{3}{c}{ Classe } \\
\cline { 2 - 4 } Parâmetros (\%) & $\mathbf{1 0 0 - 2 0 0 g}$ & $\mathbf{2 0 0 - 3 0 0 g}$ & $\mathbf{3 0 0 - 4 0 0 g}$ \\
\hline Umidade $^{1}$ & $77,10 \pm 0,28 \mathrm{a}$ & $76,00 \pm 1,48 \mathrm{ab}$ & $73,59 \pm 2,05 \mathrm{~b}$ \\
Proteína bruta $^{\mathrm{NS}}$ & $17,82 \pm 0,78$ & $16,55 \pm 1,05$ & $16,87 \pm 3,14$ \\
Cinzas $^{\mathrm{NS}}$ & $1,07 \pm 0,36$ & $1,10 \pm 0,48$ & $1,02 \pm 0,35$ \\
Lipídeos $^{1}$ & $3,18 \pm 0,95 \mathrm{c}$ & $5,08 \pm 0,97 \mathrm{~b}$ & $7,73 \pm 0,22 \mathrm{a}$ \\
Carboidratos $^{\mathrm{NS}}$ & $0,83 \pm 0,30$ & $1,27 \pm 0,52$ & $0,79 \pm 0,44$ \\
\hline${\text { Valor calórico }(\mathrm{kcal} / 100 \mathrm{~g}){ }^{1}}^{1}$ & $103,22 \pm 6,07 \mathrm{c}$ & $117 \pm 9,20 \mathrm{~b}$ & $140,21 \pm 7,71 \mathrm{a}$ \\
\hline${ }^{1}$ Médias na mesma linha seguidas por letras distintas diferem $(\mathrm{P}<0,05)$ pelo teste de Tukey. NS: Não significativo. Dados \\
expressos em média \pm desvio padrão & &
\end{tabular}

O teor de lipídeos, em pescados, é considerado benéfico, pois os peixes possuem carne rica em ácidos graxos poli-insaturados ${ }^{(31)}$. Além disso, o teor de lipídeos influi decisivamente na performance produtiva, na vida útil dos produtos e na aceitação geral pelos consumidores ${ }^{(19)}$. Gonçalves e Cezarini ${ }^{(32)}$ verificaram no jundia $R$. quelen teor de lipídeos de $2,38 \%$, inferior ao reportado no atual estudo. Melo et al. ${ }^{(33)}$ avaliaram o efeito da alimentação na composição química da carcaça do jundiá $R$. quelen e observaram valores que variaram de 12,38 a 15,09\% para a proteína bruta; 2,76 a 10,39\% 
para lipídeos; 2,13 a 2,24\% para cinzas e 70,1 a 73,16\% para umidade, valores próximos aos obtidos no atual experimento.

Os valores de composição centesimal dos peixes podem variar de acordo com a época do ano, fase fisiológica, espécie, hábito alimentar e também conforme os alimentos fornecidos aos animais. Nesse sentido, Losekann et al. ${ }^{(25)}$ observaram que os filés de jundiás $R$. quelen alimentados com dietas contendo óleo de arroz, canola ou soja, embora não apresentassem diferenças significativas nos teores de umidade, proteína bruta e cinzas, apresentaram diferentes teores de lipídeos, de acordo com o tipo de óleo administrado na ração. No presente estudo, observou-se que as diferentes classes de peso não foram determinantes para que os animais tivessem o teor de proteína bruta corporal modificada, apresentando valor médio de $17,08 \%$.

O conteúdo de carboidratos nos filés não diferiu entre as classes de peso, com valores similares aos encontrados por Caula et al. ${ }^{(34)}$, que observaram carboidratos variando entre 0,3 a 2,0\% para o pargo marinho (Lutjanus purpureus), a tilápia do Nilo, (O. niloticus), o curimatã (Prochilodus cearensise) e a sardinha (Triportheus angulatus).

Observou-se que o valor calórico dos filés de $R$. voulezi foi significativamente maior nos peixes de 301 a 400g, com média de 140,21 kcal/100 g. Filés de curimatã (P. cearensise) apresentaram valor calórico de 108,4 kcal/100 $\mathrm{g}^{(34)}$, enquanto em postas de tambaqui (Colossoma macropomum) foi encontrado $96,54 \mathrm{kcal} / 100 \mathrm{~g}^{(35)}$. Tais valores corroboram o fato de o pescado ser considerado alimento de baixo valor calórico ${ }^{(34)}$.

Estudos acerca do processamento de espécies de peixes menos conhecidas, como é o caso do jundiá $R$. voulezi, são interessantes do ponto de vista econômico, tanto para as indústrias de beneficiamento como para os produtores que podem trabalhar com as espécies regionais, mais resistentes ao clima e com possibilidade de cultivo em tanques-rede em águas de domínio público. O jundiá $R$. voulezi é um peixe com bom potencial para industrialização, por apresentar rendimentos do processamento similares entre as classes de peso estudadas. As diferenças observadas quando avaliados os rendimentos entre diferentes sexos são atribuídas às características intrínsecas de jundiás macho e fêmea.

\section{Conclusão}

Concluiu-se que os rendimentos do processamento do jundiá Rhamdia voulezi com massa entre $100 \mathrm{e}$ $400 \mathrm{~g}$ são iguais. Os machos possuem maior rendimento da carcaça em relação às fêmeas e os peixes de 301 a 400 g apresentam maior teor de lipídeos e maior valor calórico no filé.

\section{Referências}

1.Baumgartner G, Pavanelli CS., Baumgartner D, Bifi AG, Debona T, Frana VA. 1st ed. (2012). Peixes do baixo rio Iguaçu. Maringá: EDUEM; 2012, 203 p. Portuguese.

2.Diemer O, Neu, DH, Bittencourt F, Signor A, Boscolo WR, Feiden A. Eugenol como anestésico para jundiá (Rhamdia voulezi) em diferentes pesos. Semina: Ciências Agrárias. 2012;33(4):1495-1500. Portuguese.

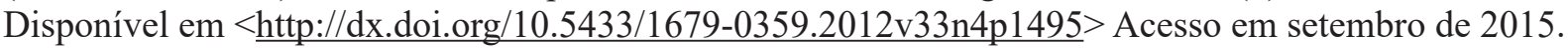

3.Reis ES, Feiden A, Signor A, Zaminhan M, Finkler JK, Boscolo WR. Suplementação de vitamina C na dieta para larvas de jundiá Rhamdia voulezi. Ciência Animal Brasileira. 2001;12(1):83-89. Portuguese. Disponível em $<$ http://dx.doi.org/10.5216/cab.v12i1.10560 $>$ Acesso em setembro de 2015.

4.Reis ES, Feiden A, Boscolo WR, Freitas JMA, Zaminhan M, Mahl I. Processamento da ração no desempenho de juvenis de jundiá (Rhamdia voulezi) cultivados em tanques-rede. Ciência Animal Brasileira, 2012;13(2):205-

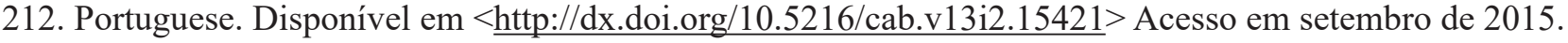

5.Diemer O, Graciano TS, Boscolo WR, Feiden A, Signor A, Signor AA. (2013). Vitamina B12 na qualidade do sêmen de jundiás, Rhamdia voulezi criados em tanques-rede. Agrarian. 2013;6(22): 500-504. Portuguese. Disponível em $<$ http://www.periodicos.ufgd.edu.br/index.php/agrarian/article/view/2287/1601 $>$ Acesso em 
setembro de 2015.

6.Diemer O, Bittencourt F, Barcellos LG, Boscolo WR, Feiden A, Romagosa E. Lysine in the diet of Rhamdia voulezi male broodstocks confined in net cages. Aquaculture. 2014; 434: 93-99. Portuguese. Disponível em $<$ http://dx.doi.org/10.1016/j.aquaculture.2014.07.029> Acesso em setembro de 2015.

7.Pessini JE, Moro EB, Fernandes DRA, Signor A, Boscolo WR, Feiden A. Vitamina A em dietas para larvas de jundiá Rhamdia voulezi. Acta Iguazu, 2014; 3(1): 28-36. Portuguese. Disponível em: http://e-revista.unioeste. br/index.php/actaiguazu/article/view/9610/7061> Acesso em setembro de 2015.

8.Basso L; Ferreira MW, Silva AR. Efeito do peso ao abate nos rendimentos dos processamentos do pacu (Piaractus mesopotamicus). Arquivo Brasileiro de Medicina Veterinária e Zootecnia. 2011; 63(5): 1260-1262. Portuguese. Disponível em $<$ http://dx.doi.org/10.1590/S0102-09352011000500033 > Acesso em setembro de 2015.

9.Galvão JA, Margeirsson S, Garate C, Viðarsson JR, Oetterer M. Traceability system in cod fishing. Food control. 2010; 21(10): 1360-1366. Disponível em $<$ http://dx.doi.org/10.1016/j.foodcont.2010.03.010 $>$ Acesso em setembro de 2015 .

10.Freato TA, Freitas RTF, Santos VB, Logato PVR, Viveiros ATM. Efeito do peso de abate nos rendimentos do processamento da piracanjuba (Brycon orbignyanus, VALENCIENNES, 1849). Ciência e Agrotecnologia. 2005; 29(3): 676-682. Portuguese. Disponível em <http://dx.doi.org/10.1590/S1413-70542005000300024> Acesso em setembro de 2015.

11.Souza ML; Maranhão TCF. Rendimento de carcaça, filé e subprodutos da filetagem da tilápia do Nilo, Oreochromis niloticus (L), em função do peso corporal. Acta Scientiarum Animal Sciences. 2001; 23(4): 897901. Portuguese. Disponível em $<$ http://dx.doi.org/10.4025/actascianimsci.v23i0.2643 $>$ Acesso em setembro de 2015.

12.Gomiero JSG, Ribeiro PAP, Ferreira MW, Logato PVR. Rendimento de carcaça de peixe matrinxã (Brycon cephalus) nos diferentes cortes de cabeça. Ciência e Agrotecnologia. 2003; 27(1): 211-216. Portuguese. Disponível em $<$ http://dx.doi.org/10.1590/S1413-70542003000100027> Acesso em setembro de 2015.

13.Carneiro PCF, Mikos JD, Bendhac F, Ignácio SA. Processamento do jundiá Rhamdia quelen: rendimento de carcaça. Revista Acadêmica: ciências agrárias e ambientais. 2004; 2(3): 11-17. Portuguese. Disponível em: http://www2.pucpr.br/reol/pb/index.php/academica?dd1=927\&dd99=view\&dd98=pb $>$ Acesso em setembro de 2015.

14. Reidel A, Romagosa E, Feiden A, Boscolo WR, Coldebella A, Signor AA. Rendimento corporal e composição química de jundiás alimentados com diferentes níveis de proteína e energia na dieta, criados em tanques rede. Revista Brasileira de Zootecnia. 2010; 39(2): 233-240. Portuguese. Disponível em < http://dx.doi.org/10.1590/ S1516-35982010000200001 > Acesso em setembro de 2015.

15.Bombardelli RA, Sanches EA. Avaliação das características morfométricas corporais, do rendimento de cortes e composição centesimal da carne do armado (Pterodoras granulosus). Boletim do Instituto de Pesca. 2008; 34(2): 221-229. Portuguese. Disponível em $<$ ftp://ftp.sp.gov.br/ftppesca/34_2_221-229.pdf $>$ Acesso em setembro de 2015.

16.Burkert D, Andrade DR, Sirol RN, Salaro AL, Rasguido JEA, Quirino CR. Rendimentos do processamento e composição química de filés de surubim. Revista Brasileira de Zootecnia. 2008; 37(7): 1137-1143. Portuguese.

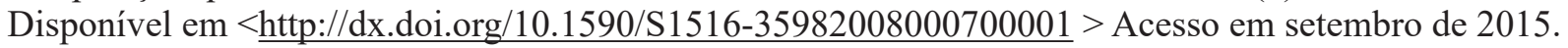

17.Basso L, Ferreira MW, Silva AR. Efeito do peso ao abate nos rendimentos dos processamentos do pacu (Piaractus mesopotamicus). Arquivo Brasileiro de Medicina Veterinária e Zootecnia. 2011; 63(5): 1260-1262. Portuguese. Disponível em $<$ http://dx.doi.org/10.1590/S0102-09352011000500033 > Acesso em setembro de 2015.

18.Britto ACPD, Rocha CB, Tavares RA, Fernandes JM, Piedras SRN, Pouey JLOF. Rendimento corporal e composição química do filé da viola (Loricariichthys anus). Ciência Animal Brasileira. 2014; 15(1): 38-44. Portuguese. Disponível em $<$ http://dx.doi.org/10.5216/cab.v15i1.21673 $>$ Acesso em setembro de 2015.

19.Souza MLR, Macedo-Viegas EM, Zuanon JAS, Carvalho MRB, Goes ESR. Processing yield and chemical composition of rainbow trout (Oncorhynchus mykiss) with regard to body weight. Acta Scientiarum. Animal Sciences. 2015; 37(2): 103-108. Disponível em <http://dx.doi.org/10.4025/actascianimsci.v37i2.24165> 
Acesso em setembro de 2015.

20.INPE. Instituto Nacional de Pesquisas Espaciais. CBERS, Satélite Sino-brasileiro de Recursos Terrestres. Imagem de Satélite de Boa Vista da Aparecida/PR, 2009. Satélite CBERS-2B, Data de passagem, 04 abr. 2009, Sensor HRC, Altura: 6894. Largura: 6398, Formato: Tiff, Res. 20 m. Portuguese. Disponível em $<\underline{\text { http://www. }}$ dgi.inpe.br/CDSR//> Acesso em setembro de 2010.

21.AOAC. Association of Official Analytical Chemistries. Official methods of analysis of the AOAC. 18 st ed. Gaithersburg: AOAC International; 2005. 1526p.

22.Souci SW, Fachman H, Kraut E. Foods Composition and Nutrition Tables. 6 st ed. Stuttgart: Medpharm Scientific Publishers; 2000. 1182p.

23.Melo JFB, Neto JR, Silva JHS, Trombetta CG. Desenvolvimento e composição corporal de alevinos de jundiá Rhamdia quelen alimentados com dietas contendo diferentes fontes de lipídeos. Ciência Rural. 2002; 32(2): 323-327. Portuguese. Disponível em $<$ http://dx.doi.org/10.1590/S0103-84782002000200023 $>$ Acesso em setembro de 2015.

24.Souza MLR, Lima S, Furuya WM, Pinto AA, Loures BTRR, Povh JA. Estudo de carcaça do bagre africano (Clarias gariepinus) em diferentes categorias de massa. Acta Scientiarum Animal Sciences. 1999; 21(3): 637-

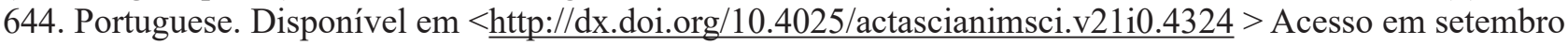
de 2015.

25.Losekann ME, Radünz Neto J, Emanuelli T, Pedron FA, Lazzari R, Bergamin GT, Corrêia V, Simões RS. Alimentação do jundiá com dietas contendo óleos de arroz, canola ou soja. Ciência Rural. 2008; 38(1): 225-230. Portuguese. Disponível em $<$ http://dx.doi.org/10.1590/S0103-84782008000100036 $>$ Acesso em setembro de 2015 .

26.Gomes, LC, Golombieski, JI, Gomes, ARC, Baldisserotto, B. Biologia do jundiá Rhamdia quelen (Teleostei, Pimelodidae). Ciência Rural, v.30, n. 1, p. 179-185, 2000. Portuguese. Disponível em <http:// dx.doi.org/10.1590/S0103-84782000000100029 > Acesso em setembro de 2015.

27.Naeem M, Salam A. Proximate composition of fresh water bighead carp, Aristichthys nobilis, in relation to body size and condition factor from Islamabad, Pakistan. African Journal of Biotechnology. 2010; 9(50): 86878692. Disponível em $<$ http://dx.doi.org/10.5897/AJB10.888 $>$ Acesso em setembro de 2015.

28.Ahmed T, Naqvi SMA, Abdullah S, Abbas K, Zakir S, Shah H, Zia MA. Comparative proximate body composition of wild captured and farm cultured Cirrhinus mrigala. Pakistan Journal of Agricultural Sciences, 2015; 52(1): 203-207. Disponível em <http://pakjas.com.pk/papers/2408.pdf $>$ Acesso em setembro de 2015.

29.Adames MS, Krause RA, Damasceno DZ, Piana PA, Oliveira JDS, Bombardelli, RA. Características morfométricas, rendimentos no processamento e composição centesimal da carne do barbado. Boletim do Instituto dePesca, 2014; 40(2):251-260. Portuguese. Disponível em <ftp://ftp.sp.gov.br/ftppesca/40_2-251-260. pdf $>$ Acesso em setembro de 2015.

30.Olopade OA. Effect of poor handling on the nutritional composition of some imported frozen fishes in Nigeria. Bulletin of University of Agricultural Sciences and Veterinary Medicine Cluj-Napoca. Food Science and Technology. 2015; 72(1): 20-26. Disponível em < $\underline{\text { http://journals.usamvcluj.ro/index.php/fst/article/ }}$ view/10893/pdf> Acesso em setembro de 2015.

31.Li G, Sinclair AJ, Li D. Comparison of lipid content and fatty acid composition in the edible meat of wild and cultured freshwater and marine fish and shrimps from China. Journal of Agricultural and Food Chemistry. 2011; 59(5): 1871-1881. Disponível em $<$ http://dx.doi.org/10.1021/jf104154q > Acesso em setembro de 2015.

32.Gonçalves AA, Cezarini R. Agregando valor ao pescado de água doce: defumação de filés de jundiá (Rhamdia quelen). Revista Brasileira de Engenharia de Pesca. 2008; 3(2): 63-79. Portuguese. Disponível em $<$ http://ppg.revistas.uema.br/index.php/REPESCA/article/view/73/72> Acesso em setembro de 2015.

33.Melo JFB, Boijink CL, Radünz Neto, J. Efeito da alimentação na composição química da carcaça do jundiá Rhamdia quelen. Biodiversidade Pampeana. 2003; 1(1) 12-23. Portuguese. Disponível em <http:// revistaseletronicas.pucrs.br/ojs/index.php/biodiversidadepampeana/article/viewFile/2461/1929 > Acesso em setembro de 2015.

34.Caula FCB, Oliveira MP, Maia EL. Teor de colesterol e composição centesimal de algumas espécies de 
peixes do estado do Ceará. Ciência e Tecnologia de Alimentos. 2008; 28(4): 959-963. Portuguese. Disponível em $<$ http://dx.doi.org/10.1590/S0101-20612008000400031 > Acesso em setembro de 2015.

35.Cartonilho MM, Jesus RS. Qualidade de cortes congelados de tambaqui cultivado. Pesquisa Agropecuária Brasileira. 2011; 46(4): 344-350. Portuguese. Disponível em $<$ http://dx.doi.org/10.1590/S0100204X2011000400002> Acesso em setembro de 2015. 